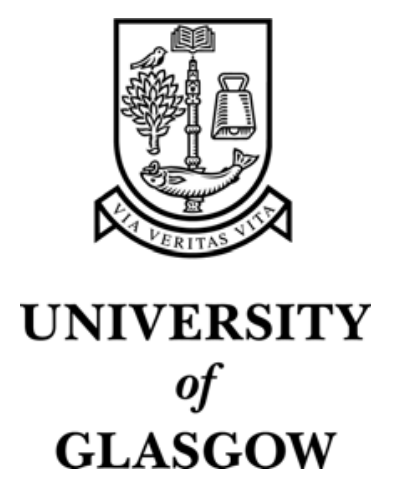

Brewster, S.A. and Murray, R. (2000) Presenting dynamic information on mobile computers. Personal Technologies 4(4):pp. 209-212.

http://eprints.gla.ac.uk/3206/ 


\title{
Presenting Dynamic Information on Mobile Computers
}

\author{
STEPHEN BREWSTER AND ROBIN MURRAY
}

Glasgow Interactive Systems Group, Department of Computing Science

University of Glasgow, Glasgow, G12 8QQ, UK

Email: stephen@dcs.gla.ac.uk Web: www.dcs.gla.ac.uk/ stephen

Tel: +44 (0)141330 4966

Fax: +44 (0) 1413304913

A problem with mobile computing devices is the output of dynamic information because their screens are small. This paper describes an experiment to investigate the use of non-speech sounds to present dynamic information without using visual display space. Results showed that nonspeech sound could be used in a simple share-dealing scenario to present a 'sound graph' of share prices. This allowed participants to reduce the workload they had to invest in share-price monitoring as they could listen to the graph whilst they worked in a share accumulation window.

\section{Introduction}

One limitation of many current Personal Digital Assistants (PDAs) and other mobile computing devices is that they present mainly static information - address books, diaries and to do lists, etc. (in a similar way to a paper organiser). The devices do not make much use of the fact that they can present dynamic data (something that paper cannot do). For example, using a PDA on a wireless network it would be possible for stock market traders to use a mobile device to keep track of stock trends when away from the trading floor. Stock prices could be sent to the device as users moved around so they could monitor the market.

Wireless networks are now becoming more common so it is possible to deliver real-time data to mobiles. The problem is how to display it given the small screens of typical devices (e.g 6 × 6cm with $160 \times 160$ pixels for the $3 \mathrm{Com}$ Palm - one of the best selling mobile computers). Visualisation techniques common on desktop displays are often unusable - they require high-resolution, large displays with many colours. For example, a common method of presenting stock trend data is a line-graph. On a mobile computer the small display and limited resolution mean that users cannot see much of the history of the trend shown in the graph - it very quickly gets scrolled off the left hand side of the screen, reducing its effective- 
ness. It is also often hard for users to see the graph if they are on the move and trying to look where they are going - visual attention is directed to moving around in the environment and not the mobile device.

One way around this problem is to use sound to present the data rather than graphics. Non-speech sound has a lot of promise because it is a way of displaying data that does not take up any screen space. The ears also have a higher temporal sensitivity than the eyes, making sound a good way to present time-varying data. In particular, SoundGraphs [3] are an alternative method for presenting line-graphs in sound for blind people. Here the pitch of a sound stream is mapped to the Yaxis of a graph and time to the $\mathrm{X}$-axis so that listeners hear a change in pitch over time. Mansur et al. [3] have shown that users are successfully able to discern the shapes of a range of different mathematical functions when they are presented as SoundGraphs. There has also been much work in the area of sonification (the equivalent of visualisation but using non-speech sound [1]) but this has not yet really been applied to mobiles. One exception is Mynatt et al. [2] who looked at how serendipitous information could be presented about activity in an office environment (using auditory icons). Users got audio information about activity within their environment based upon where they were. Mynatt et al. did not, however, carry out any evaluation to assess the effectiveness of their system. In other work at Glasgow sound has been used to improve the usability of widgets in the interfaces of mobile devices $[4,5]$ and to sonify complex menu structures to improve navigation in mobile telephones menus [6]. These studies have shown that sound can be very effective in improving usability whilst reducing the amount of visual attention needed on the device, but sound has not yet been used to provide access to time-varying, dynamic data on a handheld.

\section{Experiment}

A simple experiment was conducted to see if using SoundGraphs to present information about stock trading would be effective on a mobile. A simple share trading scenario was created with data from a month of trades from a Swiss bank. The interface to a 3Com Palm V was simulated - currently the biggest selling mobile device. The Palm has limited audio capabilities so a simulator was written in Java and tests were run on a desktop PC. This is not a mobile environment but 


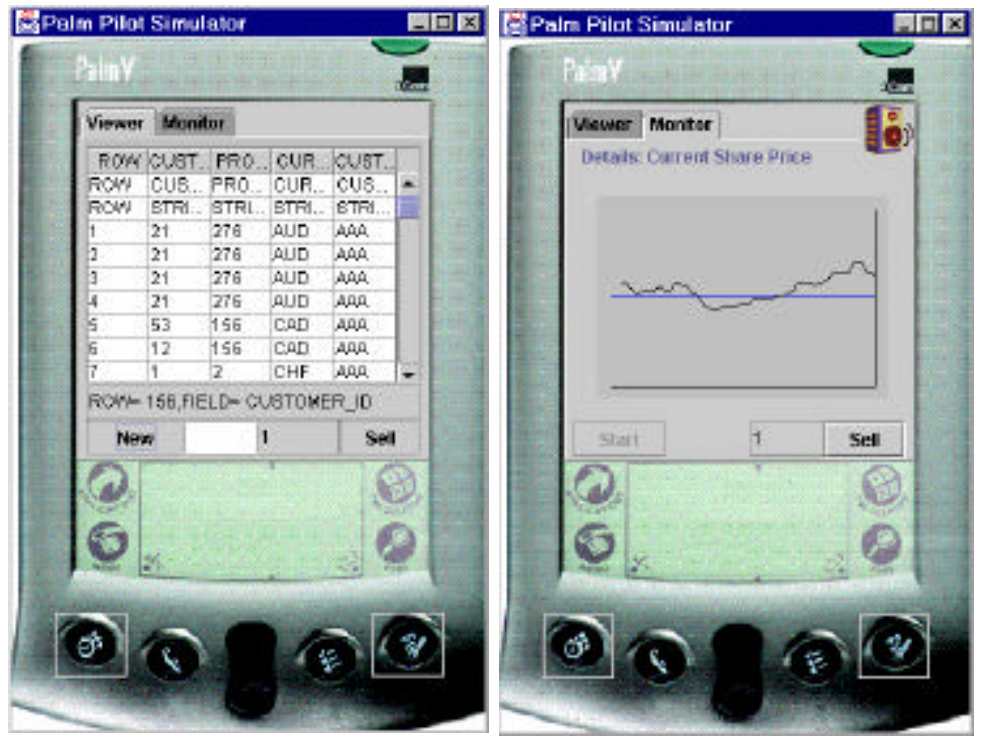

Figure 1: Screenshots of the simulator used in the experiment. The viewer window is shown on the left and the monitor window on the right.

it would give some base indication if sound could supply the dynamic information discussed here.

Experimental Task - There were two parts to the task, split into two panes of a tabbed-view on the simulator. The first part (see the Viewer window in Figure 1) required users to search a large (1000 row) table of trade information and accumulate shares to sell. Users were given a row and field heading and they then had to search (using the scrollbar) to get the value from the cell and type it in. If they did this correctly then they got one share to trade. This task required a lot of visual attention and served to distract users from the line-graph.

The Monitor window contained the line-graph, which displayed the current price of the shares. This view enabled users to sell shares, and so make a profit. The scrolling line graph was updated every second (in the simulation the values were read from a data file). The horizontal (blue) line represented the price at which the shares were bought. Any shares sold above this line resulted in a profit. To use the system participants had to perform the search tasks in the viewer window to accumulate and sell shares but also use the monitor window to know the current values of the shares to identify when it was time to sell.

Experimental design - The experiment used a counterbalanced two-condition within-groups design. In the visual condition a standard line-graph was used in the monitor window, the audio condition used the same visual line-graph and added a SoundGraph that could be heard playing continuously. Training was given before 
each condition. Workload was measured at the end of each condition. Twelve participants were used; All were students at the University of Glasgow between the ages of $20-45$. Nine were male and three female. Dependent variables were the number of times the monitor window was consulted, the time spent in this window, the profit made and subjective workload (measured using modified NASA TLX scales [7]).

Sounds used-Earcons were used to present the data. The sounds representing the data in the monitor window were similar to those from Mansur et al.[3] - the instrument used was a Saw Pad and was played on the internal MIDI synthesiser of the JavaSound package. Share values were mapped directly to MIDI notes numbers (in a similar way to Mansur et al.). Participants heard a continuous sound varying in pitch - higher pitch meant a higher price, and vice versa.

Hypotheses - The audio presentation of the monitor window should allow participants to keep a better track of the price of shares than the standard visual linegraph (as visual attention will not have to be shared across two tasks). This will be shown by fewer consultations of the monitor window, less time spent in the monitor window, more profit made and lower subjective workload.

\section{Results and Discussion}

The number of consultations of the monitor window was significantly reduced in the audio condition $\left(T_{11}=3.9, \mathrm{p}=0.002\right.$ - falling from an average of 15 lookups in the visual condition to 7 in the audio) as was the amount of time spent in the monitor window $\left(T_{11}=8.22, \mathrm{p}<0.001\right)$, confirming these hypotheses. There was, however, no difference in the number of shares bought and sold or the profit made in either of the two conditions, rejecting these hypotheses.

There was a significant reduction in the subjective workload in the auditory condition (see Figure 2). Overall average workload fell from 11.4 to $8.1\left(T_{5}=4.67\right.$, $\mathrm{p}=0.005$ ). In particular, participants rated physical demand, effort required and frustration as lower in the audio condition and their subjective performance as significantly higher $\left(T_{11}=4.46, \mathrm{p}<0.001\right)$. There was no significant difference in terms of annoyance due to the sounds but user preference was very strongly in favour of the SoundGraphs $\left(T_{11}=7.21, \mathrm{p}<0.001\right)$. These results confirm these hypotheses. 


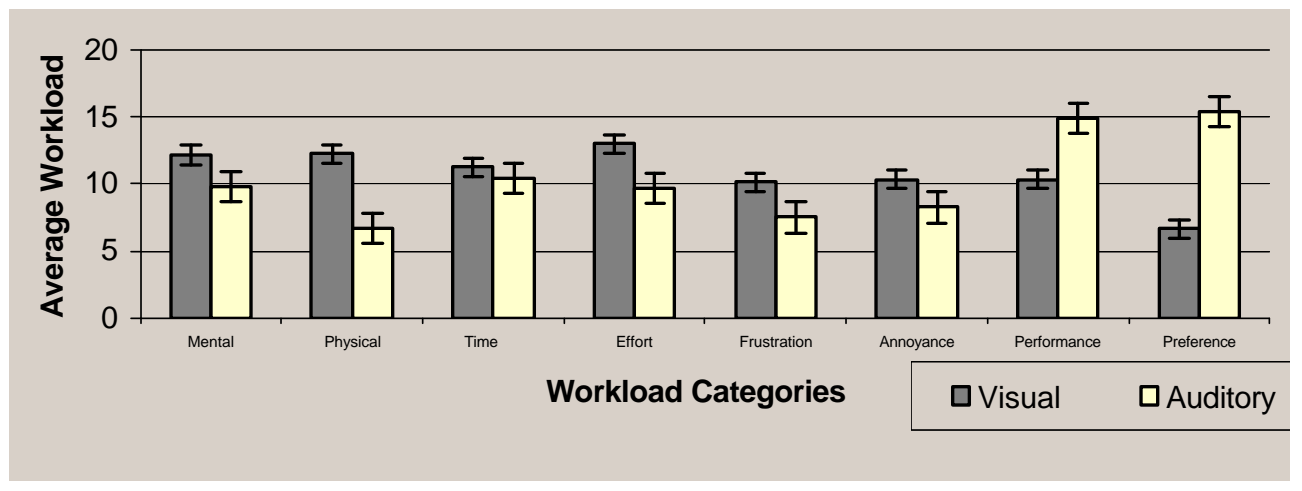

Figure 2: Average NASA TLX workload scores for all participants (Standard Error bars are shown).

The results show that the SoundGraphs were successful in reducing the frequency and amount of time that participants looked at the monitor window - they were able to do the monitoring by using the sounds whilst staying in the viewer window. Their monitoring was effective because the same profit was made in both conditions - the SoundGraphs were providing the information needed. In fact, one of the participants only looked at the monitor window once - he spent the rest of the experiment listening to the sounds to know the value of the shares.

There were strong reductions in workload with the sounds - they helped users perform the share trading tasks with a lower investment of cognitive/visual resources [7]. Participants maintained the same level of performance (and profit) in the visual condition but only at the cost of much higher workload. Reducing workload is important in situations in which users have to perform other tasks such as walking or moving around in the real world. These other tasks require many cognitive resources so that fewer can be invested in the mobile device. The results here suggest that if the share-dealing interface was used on a mobile device in a more realistic mobile environment then visual graph monitoring performance would fall. If, however, SoundGraphs were used then performance would be more robust (initial results from an evaluation undertaken whilst users were walking outside and entering data on a real Palm III have shown that significant performance and workload benefits can be gained by the addition of sound).

\section{Conclusions}

Non-speech SoundGraphs have been shown as an effective way of presenting dynamic, time-varying data on mobile computers. Their advantage is that they can be monitored whilst the user is performing another visual task and do not take up 
any of the limited amount of screen space. They also reduce the workload that a user must invest to perform the task. This is important, as the sounds would allow users to use their eyes to look where they are going and navigate around their environment whilst being able to use their mobile devices at the same time.

Acknowledgements: This work was supported by EPSRC Grant GR/L66373

\section{References}

1. Kramer, G., Eds. Auditory Display. Addison-Wesley, Reading, MA, 1994.

2. Mynatt, E.D., Back, M., Want, R. and Baer, M. Designing audio aura. In Proceedings of ACM CHI'98 (Los Angeles, CA) ACM Press Addison-Wesley, 1998, pp. 566--573.

3. Mansur, D.L., Blattner, M. and Joy, K. Sound-Graphs: A numerical data analysis method for the blind. Journal of Medical Systems, 1985; 9: 163-174.

4. Brewster, S.A. and Cryer, P.G. Maximising Screen-Space on Mobile Computing Devices. In Summary Proceedings of ACM CHI'99 (Pittsburgh, PA) ACM Press, Addison Wesley, 1999, pp. 224-225.

5. Walker, V.A. and Brewster, S.A. Spatial audio in small screen device displays. Personal Technologies, 2000; 4, 2.

6. Leplatre, G. and Brewster, S.A. Designing Non-Speech Sounds to Support Navigation in Mobile Phone Menus. In Proceedings of ICAD2000 (Atlanta, USA) ICAD, 2000, pp. 190-199.

7. Hart, S.G. and Wickens, C. Workload assessment and prediction. In Booher, H.R. (ed.) MANPRINT, an approach to systems integration, Van Nostrand Reinhold, New York, 1990, pp 257296. 